\title{
CAR-T in the clinic: drive with care
}

\author{
Rafael J. Yáñez-Muñoz ${ }^{1}$ Stephan A. Grupp ${ }^{2}$
}

Received: 22 May 2018 / Accepted: 23 May 2018

(c) Macmillan Publishers Limited, part of Springer Nature 2018

Cell and gene therapies are in the limelight after a bit of a bumpy ride. Several marketing authorisations have been granted by health authorities around the world, but perhaps the ones attracting most attention are the first USA Food and Drug Administration (FDA) gene therapy approvals for chimeric antigen receptor T-cells (CAR-T), indicated for selected cancers of the haematopoietic system. The approved CAR-T drugs are CD19-directed genetically modified autologous T-cell immunotherapies. Novartis' Kymriah (tisagenlecleucel) was approved on 30th August 2017, for the treatment of patients up to 25 years of age with B-cell precursor acute lymphoblastic leukemia (ALL) that is refractory or in second or later relapse and was the first ever US-approved gene therapy [1, 2]. Kite Pharma/Gilead's Yescarta (axicabtagene ciloleucel) was approved on 18th October 2017, indicated for the treatment of adult patients with relapsed or refractory large B-cell lymphoma after two or more lines of systemic therapy, including diffuse large Bcell lymphoma (DLBCL) not otherwise specified, primary mediastinal large B-cell lymphoma, high grade B-cell lymphoma, and DLBCL arising from follicular lymphoma [3]. Most recently, on 1st May 2018, Kymriah was also approved for the B-cell lymphoma indication [4]. Both drugs have been granted Priority Medicines (PRIME) regulatory support and are undergoing accelerated assessment by the European Medicines Agency (EMA).

The FDA-approved CAR-T drugs are autologous therapies involving the genetic modification of the patient's own cells, but have been developed with global market logistics.

Rafael J. Yáñez-Muñoz

rafael.yanez@royalholloway.ac.uk

ryanez-gt-editor@royalholloway.ac.uk

1 AGCTlab.org, Centre for Gene and Cell Therapy, Centre for Biomedical Sciences, School of Biological Sciences, Royal Holloway, University of London, Egham, UK

2 Department of Pediatrics, Children's Hospital of Philadelphia and Perelman School of Medicine, University of Pennsylvania, Philadelphia, PA, USA
So far, the EMA-approved (GSK/Orchard Therapeutics' Strimvelis, for adenosine deaminase severe combined immunodeficiency, ADA-SCID) or successfully trialled therapies for rare genetic diseases of the blood involved genetic modification of the cells at the centre delivering the therapy. In contrast, the pivotal ELIANA CAR-T clinical trial demonstrated the feasibility of delivering a therapeutic product in study sites around the world despite the cell engineering being performed at a central facility. This approach greatly facilitates expanding the number of centres where the therapy can be delivered, as there is no requirement for local cell manipulation expertise. However, given the commonality and significance of potential side effects, it is critical that treatment centres are proficient at addressing the likely complications of the therapy, especially cytokine release syndrome (CRS) and potential neurotoxicity, the latter of which may be more pronounced in CD28 costimulatory domain-containing products $[5,6]$.

Kymriah has been marketed at a list price of $\$ 475,000$ for ALL, while Kymriah and Yescarta have both been priced at $\$ 373,000$ for lymphoma, with the real expense being approximately twice as much after considering administration and follow-up. At this cost, controversy has been unavoidable. The Institute for Clinical and Economic Review (ICER) has recently released a final report on these CAR-T therapies, based on the first indication approved for each drug. The report's overall conclusion is that these therapies "provide gains in quality-adjusted and overall survival over alternative chemotherapies. With the evidence available at this time, these therapies seem to be priced in alignment with clinical benefits over a lifetime time horizon. However, the findings are sensitive to the time horizon and long-term benefit forecasting of the therapies." ICER has also made a number of policy recommendations regarding early dialogue pre-FDA approval, innovative payment models, generation of additional evidence post-treatment, settings of care and patient education (https://icer-review. org/material/car-t-final-report/).

While these CAR-T therapies have had remarkable success in clinical trials, many challenges remain, some of 
which are outlined here. Proliferation and extended persistence of the cells administered are required for therapeutic effect, but there are significant risks including CRS and neurotoxicity. Chronic B-cell aplasia is associated with long-term persistence and may require immunoglobulin replacement, but may also allow patients to remain in remission without further therapy such as transplant [2]. The CAR is composed of three modules (for antigen recognition, T-cell activation, and co-stimulation) which can be separately optimised and may lead to lower toxicity in future configurations, and the cells can be futher enhanced by engineering cytokine production. Safety switches are being developed to allow inducible activity or selective elimination of CAR-T cells. The target of the approved therapies is CD19, the B-lymphocyte antigen, but ideally therapies would target neoantigens specific to tumour-mutated genes and thus would spare normal cells. CAR-T have been approved for blood cancers, but developing therapies for solid tumour targets is of great importance and has proved more elusive to date. The cell engineering methods have relied on genetic modification through integrating retroviral and lentiviral vectors, but other technologies like transposon-mediated integration and genome editing are under development. The approved therapies are autologous, but allogeneic CAR-T approaches, which could allow off-the-shelf treatment, are also in advanced development. Finally, engineering can involve the T-cell receptor (engineered TCR T-cells) or other cells altogether (for instance, natural killer NK cells).

The WORLD ADOPT 2017 London, UK meeting, chaired by one of us (S.A.G.) and attended by the other (R. J.Y.-M.) was an opportunity to explore this expanding field and led to the production of the current special issue of Gene Therapy. The remainder of this editorial is made up of comments provided by selected speakers addressing some of the challenges in this field. Separate comments, reviews and papers explore the origins and clinical development of CAR-T technology, progress in application to solid tumours, the use of interleukin genes to enhance CAR-T cells, the risks of excessive CAR-T proliferation and the importance and possible effects of some conditioning regimes.

Novartis' Samuele Butera spoke on The globalization of CAR-T therapy: "We presented at the World ADOPT Summit on the significance of CAR-T cell therapy and the critical considerations for globalizing this treatment advance. First, it is important to understand that CAR-T therapy represents a significant step forward in treatment. Autologous CAR-T is not a pill or traditional chemotherapy; rather, it is the embodiment of personalized medicine - each dose is tailored individually to, and manufactured for, each patient using the patient's own T-cells. The therapy is produced via pioneering technology and a sophisticated manufacturing process, which starts by extracting a patient's white blood cells, including T-cells, through a specialized blood filtration process called leukapheresis. The cells are then reprogrammed to recognize and fight cancer cells and other cells expressing a specific antigen. To allow for this highly individualized treatment approach on a global scale, it is critical to have a reliable, flexible and integrated manufacturing and supply chain platform that includes a rigorous chain of identity from leukapheresis through the manufacturing process to patient infusion. The sophisticated and individualized nature of these therapies also demands specialized and trained treatment centers and ongoing site support. We must also work with payers to understand the value of this treatment approach and support safe and timely access for patients. With the historic introduction of the first FDA-approved CAR-T in August, 2017, these critical elements have come together to make a true breakthrough in innovation available to cancer patients with limited treatment options and historically poor outcomes. We continue to collaborate with University of Pennsylvania on a portfolio of CAR-T and next-generation CAR-T therapies with the goal of changing the course of cancer care."

Pfizer's Bijan Boldajipour spoke on Preclinical evaluation of allogeneic anti-B-cell maturation antigen (BCMA) chimeric antigen receptor $T$-cells: "The company has been working together with Cellectis on the development of allogeneic CAR-T cells. Genetic modifications using Cellectis' TALEN ${ }^{\circledR}$ technology allow gene knockouts of TCRa and other genes to enable the use of allogeneic cells from healthy donors. The benefits of an off-the-shelf allogeneic therapy are reduced complexity of manufacturing logistics with reduced costs, availability of the therapy to patients that cannot generate their own CAR-T, as well as potentially more healthy T-cells with better activity. UCART19 is the first allogeneic CAR-T therapy to be tested in humans, targeting CD19-positive ALL. Compassionate use has shown the potential of the therapy, phase I trials are currently being conducted. We shared our learnings from the preclinical development of allogeneic BCMA CAR-T for the treatment of multiple myeloma (MM). MM is a plasma cell disease which expresses BCMA, and targeting BCMA has shown promising activity in clinical trials using antibody-drug conjugates as well as CAR-T. We found that CAR-T candidates for BCMA were very potent killers of BCMA-expressing cells both in vitro and in vivo, but that some CAR-T would produce cytokines in the absence of targets. These CAR-T showed a reduced capacity of proliferation and maintenance of killing activity upon repeated exposure to target cells. Introduction of a rituximabsensitive safety domain did not alter T-cell activity and allowed elimination of the CAR-T by rituximab administration in animal models. Genetic modifications eliminated 
TCRa and CD52 expression, reducing graft versus host disease (GVHD) potential and enabling resistance to longterm lymphodepletion by anti-CD52 antibodies."

Adaptimmune's Helen Tayton-Martin discussed an NYO-ESO TCR trial update and advances with MAGE A4 TCR: "We highlighted commercial implications for the Adaptimmune SPEAR T-cell platform of affinity-tuned TCR T-cells, the depth and breadth of the Adaptimmune pipeline, as well as initial clinical data, including efficacy and safety of the NY-ESO program. In terms of commercialization, we discussed the implications for the cell-journey, with the patient at the core of all relevant strategies. Fully understanding the patient course will allow developers to create appropriate support tools and tailor education. We reviewed this journey in terms of implications for the payers, providers and developers. With respect to payers, it is critical to build the value story early. Ensuring the inclusion of appropriate secondary clinical trial endpoints, include quality of life and other outcome measures specifically relevant to cell therapies is important. For instance, do the current tools adequately address the unique attributes of a therapy administered via a single infusion? Are the value frameworks considering the appropriate comparative data for budget impact calculations, or assessing these therapies with enough real-world data, outside of the clinical trial setting? The developer has to understand the patient journey to arrive at TCR T-cell therapy, as well as ensuring sites are chosen with the infrastructure and expertise to accommodate and successfully administer these therapies. Developers have to address quality control of starting and ending material, cost of goods and the clinical to commercial supply chain, particularly with respect to chain of custody and identity. Lastly, it is vital to establish a cell tracking process that ensures visibility of coordination and scheduling with multiple stakeholders. In conclusion, the importance of cross-stakeholder communication was emphasized as being critical for successful TCR T-cell therapy administration and ultimate commercialization."

Steven Feldman, who at the time was Director of the National Cancer Institute (NCI) Surgery Branch Vector Production Facility, spoke on Treatment of solid cancers by adoptive cell transfer of tumor-infiltrating lymphocytes (TIL) targeting mutated tumor antigens: "The Surgery Branch has been developing cellular immunotherapy for many years. Recently we have reported on TIL recognizing unique tumor mutations mediating regression of metastatic deposits in epithelial cancers, such as cholangiocarcinoma and colon cancer. At WORLD ADOPT 2017 we presented data using whole exome and transcriptome sequence analysis of a tumor resected from a patient with treatmentrefractory metastatic breast cancer. We identified nonsynonymous somatic mutations and screened autologous TIL for recognition of these mutated antigens. Oligoclonal
TIL cultures recognizing mutated proteins were selected for adoptive cell transfer. In total, four mutated antigens were recognized by TIL and $23.1 \%$ of the infusion cell product was mutation reactive. Eight of eleven mutation-reactive clonotypes persisted more than 17 months post-cell transfer. Importantly, adoptive transfer of mutation-specific TIL mediated complete regression of the patient's metastatic breast cancer (including visceral, nodal, and subcutaneous sites) and demonstrates that adoptive transfer of TIL recognizing immunogenic mutations can be a viable treatment option for patients with breast and other solid epithelial cancers. [7]"'

John Maher, from King's College London, described a Clinical case study of intra-tumoural CAR-T cell therapy in head and neck cancer: "We have developed a CAR named T1E28z that targets the extended ErbB network. The T1E peptide is a promiscuous ErbB ligand that engages ErbB1 homo- and heterodimers and the ErbB2/3 heterodimer. CAR signalling is provided by a fused CD28 + CD3z endodomain. T1E28z is co-expressed using the SFG retroviral vector together with a chimeric cytokine receptor, $4 \alpha \beta$, that allows the selective ex vivo expansion of engineered $\mathrm{T}$ cells using IL-4. Efficacy of the resultant "T4 immunotherapy" has been demonstrated in xenograft models of established head and neck, ovarian, breast cancer and mesothelioma in vivo, without significant accompanying toxicity. The CAR can also engage mouse ErbB receptors, enabling human T-cells to kill both mouse ErbB + tumour cells and pulmonary endothelial cultures in vitro. Nonetheless, intravenous or intratumoural transfer of human T4+ T-cells into SCID Beige mice promotes tumour regression without clinical or histologically detectable toxicity. By contrast, administration of large doses of human T4+ Tcells using the intraperitoneal route elicits CRS, in a macrophage-dependent manner. These data demonstrate the existence of a therapeutic window for T4 immunotherapy in mice. To de-risk this approach in man, a phase 1 dose escalation trial has been initiated in patients with locally advanced/recurrent head and neck cancer in which intratumoural delivery without prior lymphodepletion is employed to minimise risk of toxicity. Nine patients in three dose cohorts $\left(1: 1 \times 10^{7}, 2: 3 \times 10^{7}\right.$ and $\left.3: 1 \times 10^{8}\right)$ received single intra-tumoural injections of T4. Cell product was manufactured to high specificity despite baseline lymphopenia in all patients. Treatment related adverse events (AEs) graded by common terminology criteria for adverse events (CTCAE) v4.0 were all grade 2 or below. No dose limiting toxicities (DLTs) occurred. Frequent AEs were injection lesion swelling, tumour pain and fatigue. In all three patients in cohort 3 grade 1 chills occurred within $24 \mathrm{~h}$. One patient in cohort 3 had an isolated fever within $24 \mathrm{~h}$. All patients experienced a self-limiting rise in C-reactive protein (CRP). Flow cytometric analysis of the presence of T4 
cells in the circulation was negative across cohorts. Five of the 9 patients have died from disease progression. Disease control rate (DCR) was $44 \%$ with all three patients in cohort 3 achieving stable disease despite rapidly progressing tumours on trial entry. Since the presentation, a further four patients have been treated at doses of up to 1 billion CAR T-cells, all of whom have achieved stable disease without dose-limiting toxicity."

Hans-Guido Wendel, Memorial Sloan Kettering Cancer Center, spoke on how CAR-T micro-pharmacies can restore extrinsic tumor suppression: "We identified the interactions between Herpesvirus entry mediator (HVEM, also known as tumor necrosis factor receptor superfamily member 14 , TNFRSF14) and B-lymphocyte and T-lymphocyte associated (BTLA, also known as CD272) as a major mechanism of tumor suppression in lymphoma. Accordingly, the majority of human follicular lymphomas and also many DLBCLs inactivate the HVEM receptor through somatic mutations and deletions. However, a soluble form of HVEM (solHVEM) that encodes the ectodomain of the receptor has anti-lymphoma activity in vitro and in vivo. We have developed CD19-directed CAR-T cells that also produce and secrete solHVEM locally and continuously at the lymphoma site. These modified CAR-T cells, which we call "micro-pharmacies", show superior activity compared to regular, CD19-directed CAR-T cells. We propose that CAR-T cells may be broadly useful as vehicles for the targeted production and secretion of anti-tumor proteins and peptides. Such CAR-T "micro-pharmacies" may have broad applications and deliver a therapeutic activity directly to the site where it is needed. [8]"

Affimed's Thorsten Gantke described AFM13, a bispecific NK-cell engager modulating innate and adaptive immunity: "NK-cells are cytotoxic innate lymphoid cells which are critically involved in the recognition and destruction of virally-infected and malignant cells. Thus, they have been recognized as an integral component of cancer immunotherapy approaches. The natural cytotoxicity of NK-cells is frequently dysregulated in cancer and although NK-cell activation and tumor specificity can be increased using monoclonal antibodies, classical antibodydependent cell-mediated cytotoxicity (ADCC)-inducing IgGs have limitations. These include impairment by direct competition with serum IgG for target receptors as well as the comparatively high level of target antigen expression required. Affimed's tetravalent, bispecific NK-cell engagers, e.g., AFM13 (CD16A/CD30-specific, developed to treat hematologic malignancies), AFM26 (CD16A/ BCMA-specific, developed to treat multiple myeloma) and AFM24 (CD16A/EGFR-specific, developed to treat solid tumors) have been designed to overcome these limitations. The high affinity binding to both an NK-cell and a tumor cell induces target cell lysis with superior potency and efficacy when compared with monoclonal antibodies. Importantly, CD16A-directed bivalent NK-cell binding is largely unaffected by high levels of serum IgG suggesting a mode of CD16A binding that is distinct from that of classical antibody formats. Affimed has furthermore shown that CD16A-mediated stimulation sensitizes NK-cells to IL-2 and IL-15-induced proliferation and increases NK-cell "natural" cytotoxicity following re-stimulation with IL-2 or IL-15. These data provide a strong rationale for combination of CD16A-directed bispecific NK-cell engagers with these cytokines. Lastly, due to their unique mode of NK-cell engagement and high affinity for CD16A, Affimed's bispecifics may be uniquely suited to enable novel therapeutic approaches by combining it with adoptive NK-cell transfer. To explore this concept, the company has entered into a strategic collaboration with The University of Texas MD Anderson Cancer Center aimed at bringing this novel approach to patients."

As Gene Therapy showcases in this special issue, progress in cellular immunotherapies has been phenomenal. These treatments are addressing lifethreatening cancers and come with significant side effects. Funny titles involving CARs are easy to come up with but, same as when you are behind the wheel of a powerful vehicle, driving can be exhilarating and make you forget the risks involved. The challenge is learning to drive safely while making the most of the ride - for the benefit of people affected.

Acknowledgements The authors thank Samuele Butera, Bijan Boldajipour, Helen Tayton-Martin, Steven Feldman, John Maher, HansGuido Wendel and Thorsten Gantke for providing summaries of their presentations at WORLD ADOPT 2017.

\section{Compliance with ethical standards}

Conflict of interest S.A.G. receives research support and consultancy from Novartis. The remaining author declares that he has no conflict of interest.

\section{References}

1. Maude SL, Laetsch TW, Buechner J, Rives S, Boyer M, Bittencourt $\mathrm{H}$, et al. Tisagenlecleucel in children and young adults with B-cell lymphoblastic leukemia. $N$ Engl J Med. 2018;378:439-48.

2. Maude SL, Frey N, Shaw PA, Aplenc R, Barrett DM, Bunin NJ, et al. Chimeric antigen receptor $\mathrm{T}$ cells for sustained remissions in leukemia. N Engl J Med. 2014;371:1507-17.

3. Neelapu SS, Locke FL, Bartlett NL, Lekakis LJ, Miklos DB, Jacobson CA, et al. Axicabtagene ciloleucel CAR T-cell therapy in refractory large B-cell lymphoma. N Engl J Med. 2017;377: 2531-44.

4. Schuster SJ, Svoboda J, Chong EA, Nasta SD, Mato AR, Anak Ö, et al. Chimeric antigen receptor $\mathrm{T}$ cells in refractory B-cell lymphomas. N Engl J Med. 2017;377:2545-54. 
5. Neelapu SS, Tummala S, Kebriaei P, Wierda W, Gutierrez C, Locke FL, et al. Chimeric antigen receptor T-cell therapyassessment and management of toxicities. Nat Rev Clin Oncol. 2018;15:47-62.

6. Teachey DT, Bishop MR, Maloney DG, Grupp SA. Toxicity management after chimeric antigen receptor $\mathrm{T}$ cell therapy: one size does not fit 'ALL'. Nat Rev Clin Oncol. 2018;5:218.
7. Zacharakis N, Chinnasamy H, Black M, Xu H, Lu Y-C, Zheng Z, et al. Immune recognition of somatic mutations leading to complete durable regression in metastatic breast cancer. Nat Med. 2018; https://doi.org/10.1038/s41591-018-0040-8.

8. Boice M, Salloum D, Mourcin F, Sanghvi V, Amin R, Oricchio E, et al. Loss of the HVEM Tumor Suppressor in Lymphoma and Restoration by Modified CAR-T Cells. Cell 2016;167:405-418. 